\title{
Self-Assembly of PZT Actuators for Micropumps With High Process Repeatability
}

\author{
Jiandong Fang, Kerwin Wang, and Karl F. Böhringer, Senior Member, IEEE
}

\begin{abstract}
In this paper, we report a novel capillary-driven self-assembly technique which proceeds in an air environment and demonstrate it by assembling square piezoelectric transducer (PZT) actuators for 28 diffuser valve micropumps on a 4-inch pyrex/silicon substrate: on the substrate, binding sites are wells of $24 \mu \mathrm{m}$ in depth and the only hydrophilic areas; on the bonding face of the PZT actuator, the central hydrophilic area is a square identical in size to the binding site, and the rim is hydrophobic; acrylate-based adhesive liquid is dispensed across the substrate and wets only the binding sites; the hydrophilic areas on the introduced PZT actuators self-align with the binding sites to minimize interfacial energies by capillary forces from the adhesive droplets; the aligned PZT actuators are pressed to contact the gold coated substrate by their rims and the adhesive is polymerized by heating to $85^{\circ} \mathrm{C}$ for half an hour, so permanent mechanical and electrical connections are established, respectively, at the center and rim of each PZT actuator. These pumps perform with high uniformity, which is indicated by a small standard deviation of their resonant frequencies to pump ethanol: the average resonant frequency is $6.99 \mathrm{kHz}$ and the standard deviation is $0.1 \mathrm{kHz}$. Compared with the conventional bonding process with highly viscous silver epoxy, this assembly method has several major advantages: highly accurate placement with self-alignment, controllable adhesive thickness, tilt free bonding, low process temperature and high process repeatability.

[1491]
\end{abstract}

Index Terms-Capillary-driven self-assembly, diffuser valve micropump, recessed binding site, interfacial energy minimization.

\section{INTRODUCTION}

$\mathbf{P}$ ZT actuators can convert electrical energy to mechanical energy with fast response and are widely used as driving elements for several types of microfluidics devices such as micropumps [1], micromixers [2], [3] and microdispensers [4], among others. Typically, piezoelectric transducer (PZT) actuators are manually mounted onto a silicon, glass, or polymer substrate with batch-fabricated microfluidic components using highly viscous silver epoxy, a slow serial process without good control of process parameters such as placement of PZT actuators, adhesive thickness and PZT actuator tilting; therefore this assembly process cannot achieve good repeatability and does not scale well to wafer level packaging.

Capillary forces have been exploited to assemble microstructures in two or three dimensions by several research groups [5]-[9]. We previously reported capillary-driven self-assembly

Manuscript received January 1, 2005; revised November 11, 2005. This work was supported by NIH Center of Excellence in Genomic Science and Technology grant 1-P50-HG002360-01 and DARPA DSO award FAA9550-04-0257. Subject Editor D.-I. Cho.

The authors are with the Department of Electrical Engineering, University of Washington, Seattle, WA 98195 USA (e-mail: jdfang@ee.washington.edu; karl@ee.washington.edu).

Digital Object Identifier 10.1109/JMEMS.2006.878880 of surface mounted light emitting diodes (LEDs) in a water environment [10]: a hydrophilic substrate was patterned with hydrophobic binding sites, then adhesive liquid was dispensed across the substrate in air and wet only the binding sites when the substrate was submerged in the water, and the adhesive droplets on the binding sites attracted and aligned the introduced LEDs to minimize interfacial energies, finally the adhesive liquid was polymerized by heating for permanent bonding. This assembly technique is assumed to have the following properties: 1) microcomponents to be assembled are soaked in an aqueous environment for quite a long time; 2) the adhesive volume on each binding site cannot be effectively controlled; 3) part tilting is hard to avoid [11]; 4) bonding strength is poor because the binding site is hydrophobic with low interfacial energy, which will be demonstrated by a debonding test in Section IV; 5) electroplating is required to establish electrical connections from the substrate to the LEDs. To achieve good and repeatable performance for PZT driven devices, PZT actuators should be bonded with the following requirements: strong and tilt free bonding, accurate placement and precisely controlled adhesive thickness. These requirements indicate the above method to assemble LEDs cannot be the optimal choice to bond PZT actuators.

We have developed a novel method to bond PZT actuators with a well-controlled self-assembly process. This method is a parallel process in an air environment and provides controlled adhesive thickness, highly accurate placement and tilt free bonding. The bonding adhesive [6] (an acrylate-based liquid) has very low viscosity and significant surface tension to align PZT actuators, and can be polymerized by heating to $85^{\circ} \mathrm{C}$ for about half an hour. The adhesive only bonds PZT actuators mechanically because it is not electrically conductive. We use a design strategy with precisely recessed binding sites to establish mechanical bonding and electrical bonding, respectively, at the center and rim of a PZT actuator. This technique is demonstrated by mounting PZT actuators for 28 diffuser valve micropumps on a 4-inch pump substrate.

\section{CAPILlaRY-Driven SElF-AsSEMBly MECHANISM}

\section{A. Interfacial Energy Minimization}

A schematic top view of a part captured but not yet aligned by a binding site via a layer of adhesive liquid film is shown in Fig. 1: $P$ stands for the bottom surface of a part, and $B$ for a binding site, and $S$ for the binding site background. For most capillary-driven self-assembly processes, $B$ and $P$ are of an identical shape. Our self-assembly process in an air environment has the following properties: 1) $P$ and $B$ are hydrophilic; 2) $S$ is hydrophobic; 3) adhesive liquid exclusively and completely 


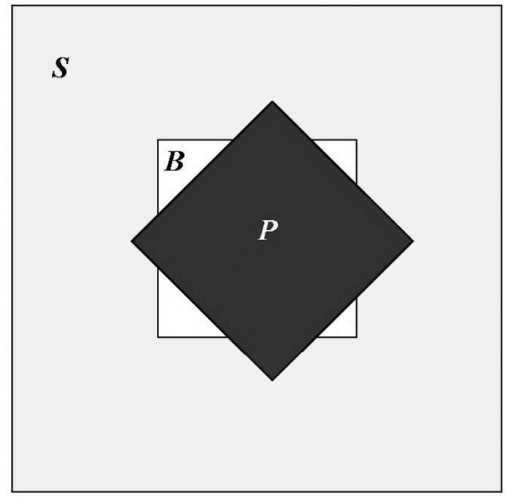

Fig. 1. A schematic top view of a part $P$ captured but not yet aligned by a hydrophilic binding site $B$ with hydrophobic background $S$ via a layer of adhesive liquid film.

wets hydrophilic surfaces $P$ and $B$, i.e., the small gap between $P$ and $S$ is also wetted. To estimate the total interfacial energy $E$ of the arrangement in Fig. 1, we observe that the adhesive film thickness is much less than the lateral size of $P$ or $B$, and that interfacial energy is proportional to interfacial area. Therefore, the interfacial energy at the sidewalls of the adhesive film can be neglected, and total interfacial energy of this system can be approximated with

$$
\begin{aligned}
E=\gamma_{f p}|P|+\gamma_{f a} \mid B- & P \cap B\left|+\gamma_{f b}\right| B \mid \\
& +\gamma_{f s}|P \cap S|+\gamma_{s a}|S-P \cap S|
\end{aligned}
$$

Where $\gamma_{i j}$ is interfacial energy between material $i$ and material $j$, and subscript $f$ stands for adhesive film, $a$ for air, and $p / b / s$ for surface $P / B / S .|A|$ denotes the area of a surface $A$. The operators " $\cap$ " and "-_" are respectively geometric intersection and difference. The terms of the right hand side of (1) come respectively from the following interfaces: complete adhesive wetting of $P$; part of adhesive on $B$ that is exposed to air; complete adhesive wetting of $B$; adhesive wetting of the part of $S$ that is overlapped by $P$; the part of $S$ exposed to air.

Overlap area between $P$ and $S$ can be defined by: $|P \cap S|=$ $|P|-|P \cap B|$. By replacing $|P \cap S|$ in (1), we can get

$$
\begin{aligned}
E= & \gamma_{f p}|P|+\gamma_{f a}(|B|-|P \cap B|)+\gamma_{f b}|B| \\
& +\gamma_{f s}(|P|-|P \cap B|)+\gamma_{s a}(|S|-|P|+|P \cap B|)
\end{aligned}
$$

In (2), $|P \cap B|$ is the only variable factor depending on the position of $P$ relative to $B$. By collecting the constant and the variable terms, we can rewrite (2) as

$$
\begin{aligned}
E= & {\left[\gamma_{f p}|P|+\gamma_{f a}|B|+\gamma_{f b}|B|\right.} \\
& \left.\quad+\gamma_{f s}|P|+\gamma_{s a}(|S|-|P|)\right] \\
& -\left(\gamma_{f a}+\gamma_{f s}-\gamma_{s a}\right)|P \cap B| \\
= & C_{1}-C_{2}|P \cap B|
\end{aligned}
$$

where $C_{1}=\gamma_{f p}|P|+\gamma_{f a}|B|+\gamma_{f b}|B|+\gamma_{f s}|P|+\gamma_{s a}(|S|-|P|)$ and $C_{2}=\gamma_{f a}+\gamma_{f s}-\gamma_{s a}$ are constants. Usually, $\gamma_{s a}$ is much smaller than $\gamma_{f a}$, which leads to a positive $C_{2}$. Therefore, the total interfacial energy is a linear function of the overlap area $|P \cap B|$ with a minimum when $P$ and $B$ exactly align.

\section{B. Self-Alignment of Rectangular Parts}

According to (3), the overlap area between a part and a binding site during self-alignment of the part provides a scalar value that can be used to find a final stable state. In practice, most microparts are rectangular because of easy mechanical dicing along straight lines. During the following discussions, only rectangular parts are considered, but our analysis generalizes directly to parts of arbitrary shapes. Self-alignment motion of a part is a combination of translation and rotation. For simplicity, we visualize the overlap area $|P \cap B|$ for translation and rotation separately: for translation [see Fig. 2(a)], the result shows a unique maximum overlap area when the part is exactly aligned with the binding site; for rotation [see Fig. 2(b)], the result indicates that the number of maximum overlap states or minimum energy states depends on the width-to-length ratio of the rectangular part: a square part has four preferred in-plane orientations with rotation angle intervals of $90^{\circ}$; as the width-to-length ratio decreases, the overlap area peaks near $90^{\circ}$ and $270^{\circ}$ shrink and become valleys when the width-to-length ratio reaches approximately $1: 1.3$, thus a typical rectangular part has two preferred in-plane orientations.

Square PZT actuators can be aligned to any of the four in-plane orientations with minimum interfacial energy during an assembly process. A PZT actuator has two large opposite faces used as two electrodes, i.e., the bonding face has only one electrode. Electrical connections to the bonding face can be established correctly no matter which in-plane orientation with minimum interfacial energy the PZT actuator takes. Connection to the opposite, outward facing electrode can be achieved, e.g., with a wire bond.

\section{Diffuser VAlve MicroPumps}

\section{A. Working Principle}

We choose diffuser valve micropumps for the demonstration of PZT self-assembly because this type of pump is a good candidate to control the transport of liquids from microliters to nanoliters [12] and the diffuser valve is well understood [13]-[15]. The diffuser valve micropump (Fig. 3) consists of a large shallow chamber, two diffuser check valves, two channels connecting the valves and the inlet/outlet holes, and the pump diaphragm is actuated by a unimorph structure-a single layer PZT bonded to the diaphragm: the PZT actuator expands and contracts in the diaphragm plane during a cycle of an ac driving voltage, which causes the diaphragm to bend upwards and downwards. A diffuser valve has lower resistance for flow from the narrow port to the wide port than the reverse flow. During a half ac cycle, the pump chamber volume increases, and more flow comes from the inlet valve; during the other half ac cycle, the pump chamber volume decreases, and more flow goes out through the outlet valve.

\section{B. Fabrication}

The silicon pump substrate is etched by two deep reactive ion etching (DRIE) processes. Photoresist AZ4620 is used as the 

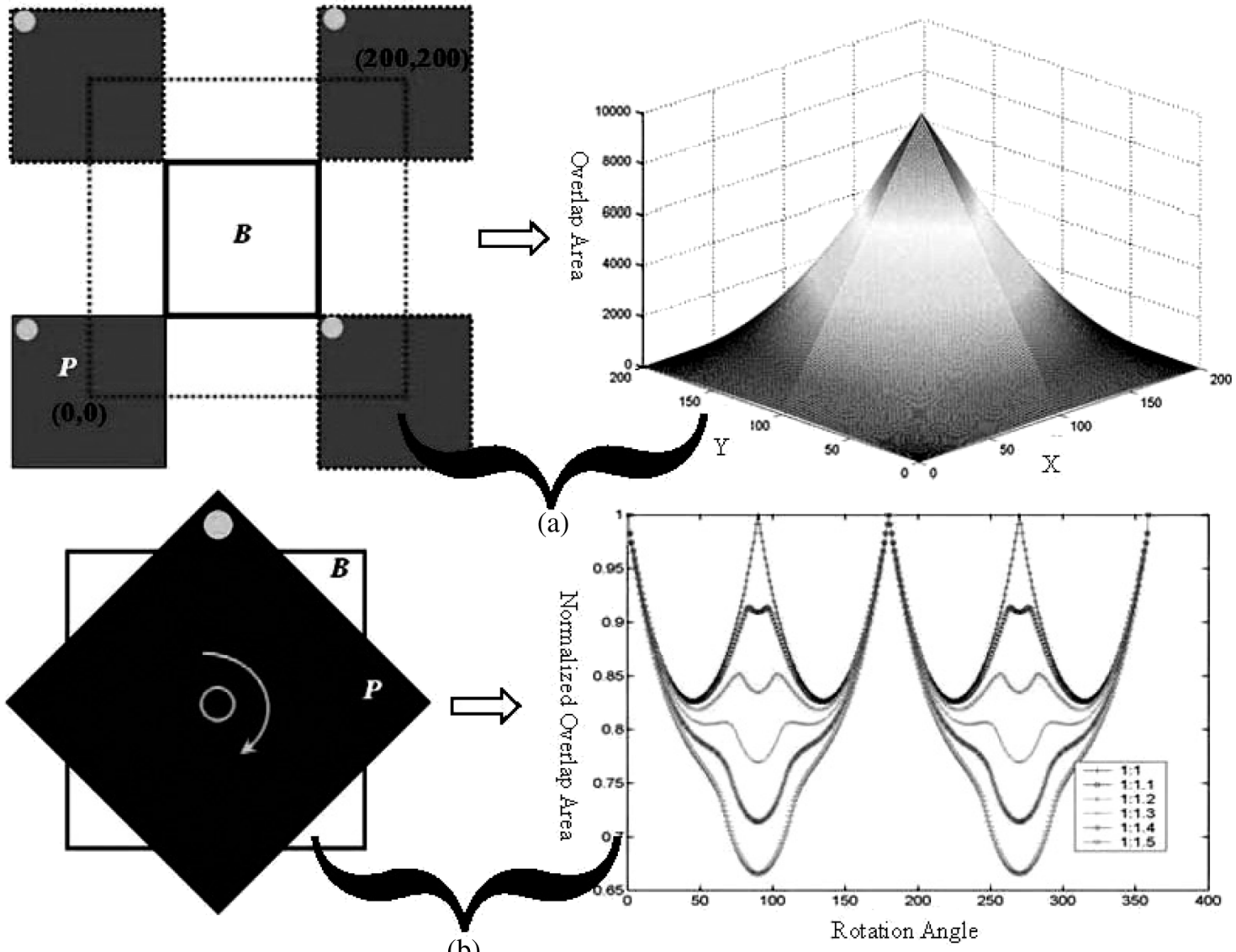

(b)

Fig. 2. Visualization of overlap area between a moving part and a binding site: (a) translation of a square part (size: $100 \times 100$ units); (b) rotation of a rectangular part with different aspect ratios. For parts that are nearly square, 4 overlap maxima exist. For more elongated rectangles (aspect ratio beyond 1:1.3) only 2 overlap maxima remain.

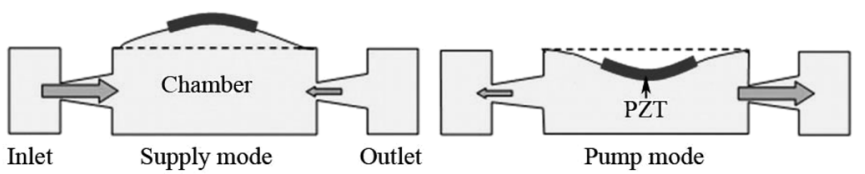

(a)

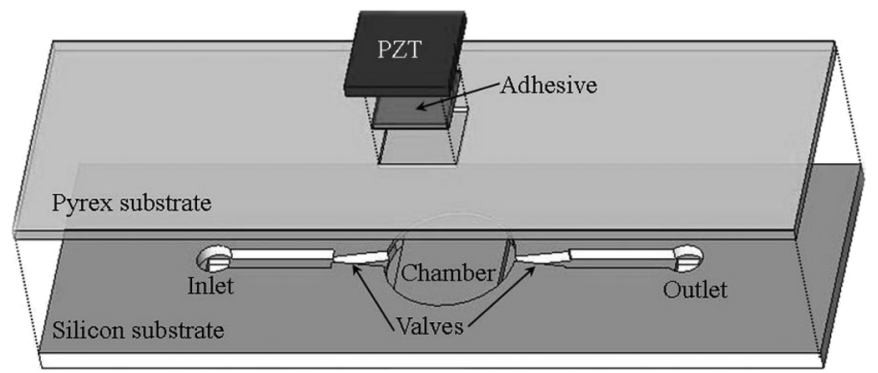

(b)

Fig. 3. Schematic views of a diffuser valve micropump: (a) supply and pump modes, net flow to the right; (b) an exploded view.

mask for each DRIE process and patterned by photolithography with the following steps: 1) singe the substrate on a $110^{\circ} \mathrm{C}$ hotplate for $1 \mathrm{~min}$; 2) spincoat primer P10 and photoresist AZ4620 subsequently at the speed of $2200 \mathrm{rpm}$ for $30 \mathrm{~s}$ (AZ4620 thickness is about $8 \mu \mathrm{m})$; 3) prebake AZ4620 on a $110{ }^{\circ} \mathrm{C}$ hotplate for $3 \mathrm{~min}$; 4) expose AZ4620 with a mask for $30 \mathrm{~s}$ to
$10 \mathrm{~mW} / \mathrm{cm}^{2}$ UV light; 5) develop AZ4620 in a solution of AZ400K: DI water $=1: 4$ for $2 \mathrm{~min}$, then rinse the substrate in DI water and dry it with a $\mathrm{N}_{2}$ gun; 6) hardbake AZ4620 on a $110^{\circ} \mathrm{C}$ hotplate for $15 \mathrm{~min}$. The first DRIE is to etch pump chambers, which are $5 \mathrm{~mm}$ in diameter and $24 \mu \mathrm{m}$ in depth. The large and shallow features of the chambers make spincoating of another layer of AZ4620 feasible, producing a sufficiently conformal coating of photoresist over this step feature. The second DRIE is used to etch diffuser valves and channels with a total depth of $275 \mu \mathrm{m}$. After each DRIE is done, the photoresist is stripped with the following process: soak in $60^{\circ} \mathrm{C}$ EKC solution for 10 minutes and in $75{ }^{\circ} \mathrm{C} \mathrm{AZ300T} \mathrm{solution} \mathrm{for} 5 \mathrm{~min}$, and then rinse in DI water and dry with a $\mathrm{N}_{2}$ gun. A laser cutter (Model 4410, ESI, OR) is run in a raster mode to drill $0.8 \mathrm{~mm}$ square inlet and outlet holes to the back side of the substrate (Fig. 4).

A $500-\mu \mathrm{m}$-thick Pyrex wafer is anodically bonded to the etched silicon substrate [see Fig. 5(a)]. Before bonding, both the silicon substrate and the pyrex wafer are cleaned with the following process: the silicon substrate is dipped in buffered oxide etchant (BOE) for 1 minute to remove native oxide, and soaked in a Nano-strip solution (Rockwood Electronic Materials, CA, USA) for $15 \mathrm{~min}$ together with the pyrex wafer to strip organic residues, then both wafers are soaked in flowing DI water for about $5 \mathrm{~min}$ and dried in a spin dryer. After thorough cleaning 


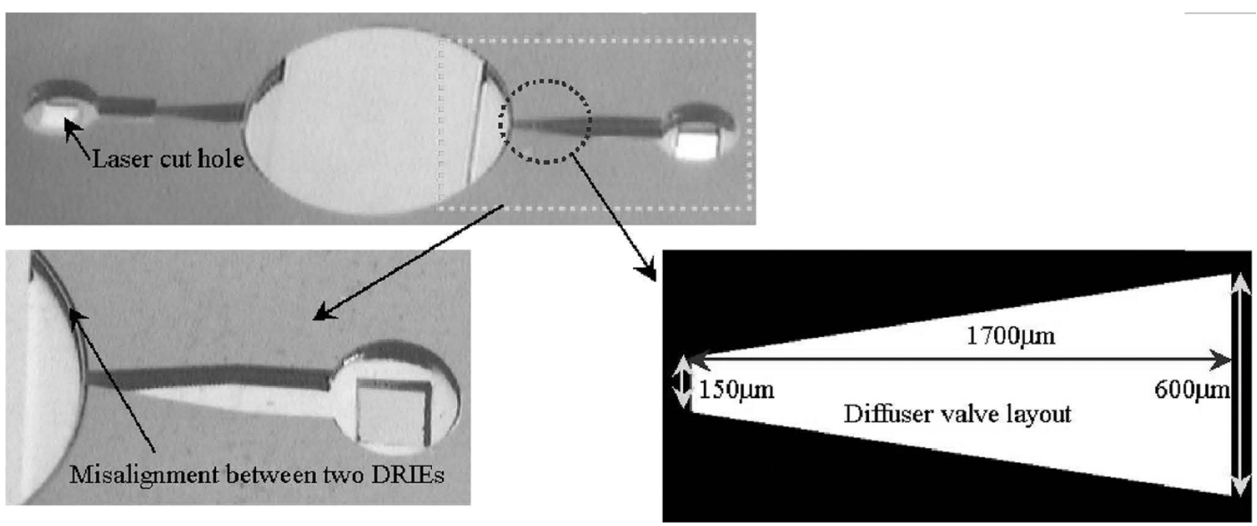

Fig. 4. Microscope images of DRIEed silicon substrate and the diffuser valve layout.

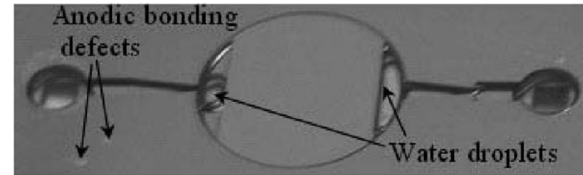

(a)

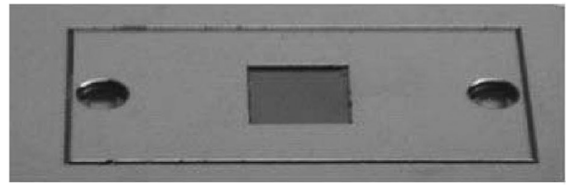

(b)

Fig. 5. (a) An optical image of the anodically bonded pyrex/silicon stack (water droplets are intentionally introduced to the channels to indicate the pyrex capping); (b) an optical image of patterned Au covering the pyrex surface (the square receptor site at the center of the pump chamber is recessed by $24 \mu \mathrm{m}$, and two circular openings are used for later alignment with fluid interconnects on the pumping test stage, see Fig. 13).

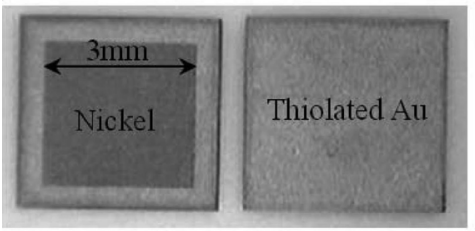

(a)

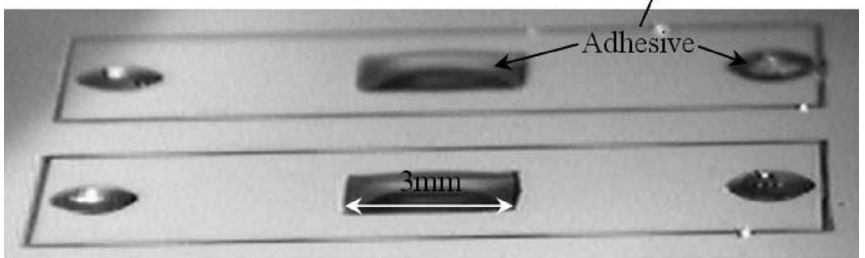

(c)
Fig. 6. Wetting property of PZT parts and the pump substrate: (a) top and bottom views of a PZT part; (b) adhesive liquid wets only the nickel bonding area on the PZT part; (c) adhesive liquid wets the recessed pyrex binding sites on the pump substrate.

both wafers are transferred to a $250{ }^{\circ} \mathrm{C}$ hotplate and heated for about $2 \mathrm{~min}$, and then immediately transferred to a $500{ }^{\circ} \mathrm{C}$ hotplate to be baked for $5 \mathrm{~min}$. Two-stage heating prevents wafers from cracking by a significant thermal stress due to sudden change of temperature. Then the pyrex wafer is placed on the silicon substrate and a dc voltage of $1200 \mathrm{~V}$ is applied to the stack with the cathode electrode probe touching the pyrex surface, so that the pyrex wafer is permanently bonded to the silicon substrate.

To get a thinner pump diaphragm, HF (49\%wt) is used to etch pyrex. A piece of dicing blue tape is attached to the back side of the pump substrate to cover inlet and outlet holes, which keeps the pump cavities clean from the etchant. The pyrex wafer is thinned down to $230 \mu \mathrm{m}$ in thickness within $45 \mathrm{~min}$. Finally the substrate is cleaned in DI water and dried by a $\mathrm{N}_{2}$ gun, and the blue tape is manually peeled off.

\section{PZT SELF-ASSEMBLY}

\section{A. Hydrophilic Recessed Binding Sites}

The binding sites for self-alignment of the PZT actuators are designed as recessed hydrophilic wells in a hydrophobic surface [see Fig. 5(b)]. These shallow wells contain the adhesive liquid that drives the assembly, and are slightly smaller than the PZT actuators, such that the well rims can make electrical contacts with the PZT actuators. A layer of $\mathrm{Cr} / \mathrm{Au}$ (50/ $1000 \AA$ ) is deposited on the pyrex surface of the bonded pump substrate and patterned by an etching process. The etching mask is photoresist AZ1512 patterned by photolithography to expose the 3-mm-square binding sites directly above the centers of the pump chambers. After $\mathrm{Cr} / \mathrm{Au}$ etching, pyrex in the binding sites is etched by HF (49\%wt) until $24 \mu \mathrm{m}$ deep wells are formed, and the etching rate is about $6.75 \mu \mathrm{m} / \mathrm{min}$.

\section{B. Fabrication of PZT Parts}

PZT actuators are fabricated from a piece of $2.85^{\prime \prime} \times 2.85^{\prime \prime} \times$ $0.0075^{\prime \prime}$ PZT sheet (T107-H4E-602, Piezo Systems, Cambridge, MA) which is coated with $\sim 3000 \AA$ thick nickel on both sides. Following are the fabrication steps: 1) AZ1512 is spincoated and UV exposed with a mask protecting 3-mm square areas used for later bonding (space between two squares is $1 \mathrm{~mm}$ ), after developing only the square bonding areas are coated with AZ1512; 2) Cr/Au (50/1000 A) is deposited on both sides of the PZT sheet by two thermal evaporations; 3 ) the PZT sheet is rinsed in acetone to strip AZ1512 along with $\mathrm{Cr} / \mathrm{Au}$ on it, then the square bonding areas are exposed [see Fig. 6(a)]; 4) the PZT sheet is diced by a laser cutter into 4-mm square parts with the $3 \mathrm{~mm}$ square bonding area at the center; 5) the diced parts are cleaned with acetone, IPA and DI water in sequence inside a sonicator. Finally the cleaned parts are collected to a piece of filter paper and dried by baking on a $100{ }^{\circ} \mathrm{C}$ hotplate for $5 \mathrm{~min}$. 


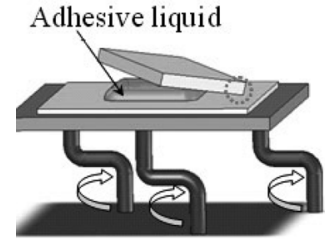

(a)

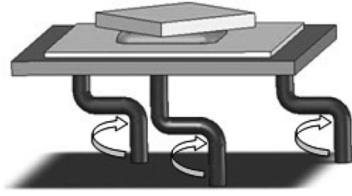

(b)

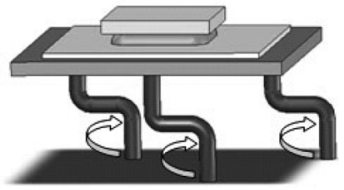

(c)

Fig. 7. Schematic diagrams for orbital shaking assisted self-assembly: (a) a part sticks to the substrate by its right corner; (b) a temporarily left-directed centrifugal force from the orbital shaking releases and balances the tilted part; (c) the balanced part immediately self-aligns to the binding site, and keeps its alignment at the minimum interfacial energy state when the centrifugal force changes direction.

TABLE I

Contact Angles of WATER AND THE AdHesive LiQUid on DifFERENT SURFACES

\begin{tabular}{|c|c|l|c|l|}
\hline & Pyrex & $\begin{array}{l}\text { Thiolated Au } \\
\text { on Pyrex }\end{array}$ & PZT & $\begin{array}{l}\text { Thiolated Au } \\
\text { on PZT }\end{array}$ \\
\hline Water & $32^{\circ}$ & $108^{\circ}$ & $40^{\circ}$ & $102^{\circ}$ \\
\hline Adhesive liquid & $4^{\circ}$ & $51^{\circ}$ & $0^{\circ} a$ & $40^{\circ}$ \\
\hline
\end{tabular}

$a_{\text {Note: }} 0^{\circ}$ indicates super high wettability, i.e., a droplet spreads quickly into a thin film on a substrate.

\section{Surface Treatment for the Pump Substrate and PZT Parts}

The pump substrate and the PZT parts are soaked overnight in a $1 \mathrm{mmol}$ alkanethiol solution (diluted in ethanol) for gold surfaces to adsorb a self-assembled monolayer (SAM). Contact angles of water and the adhesive liquid on the treated surfaces are respectively measured by a FTA200 goniometer (First Ten Angstroms, Inc.), and the results (Table I) indicate that the thiolated Au surfaces on either pyrex or PZT substrates have much less attraction for both water and the adhesive liquid than pyrex and nickel surfaces. Flushing of the adhesive liquid across the pump substrate or the bonding face of a PZT part leaves droplets only on the pyrex binding sites or nickel bonding areas on the PZT parts [see Fig. 6(b) and (c)].

\section{Orbital Shaking Assisted Self-Assembly}

This self-assembly technique exploits surface tension force to self-align PZT parts to the photolithography-defined $3 \mathrm{~mm} \times 3 \mathrm{~mm} \times 24 \mu \mathrm{m}$ hydrophilic recessed binding sites. In an air environment the acrylate-based adhesive only wets the hydrophilic areas of the recessed binding sites and nickel areas on the PZT parts (Fig. 6). Dry PZT parts are introduced manually without alignment onto the adhesive droplets on the binding sites with the exposed nickel surface facing downward. According to the capillary-driven self-assembly mechanism described in Section II, the minimum interfacial energy state can be achieved when the PZT part is aligned with the recessed binding site. But because the $4 \mathrm{~mm}$ square PZT part is larger than the $3 \mathrm{~mm}$ square binding site, the unaligned PZT parts tend to tilt and touch the substrate with their corners or edges, which causes significant friction, preventing surface tension from aligning the parts. As a remedy, an orbital shaker (Model 51300-00, Cole-Parmer Instrument Company) is used to provide centrifugal forces to the PZT parts. The centrifugal forces drag and balance the parts on the adhesive droplets temporarily so that surface tension can drive self-alignment of these PZT parts (Fig. 7). To assist self-assembly, orbital shaking introduced centrifugal force $m \omega^{2} R_{s}$ should be less than the surface tension $F_{s t}$ on the part by the adhesive, therefore the shaking speed $\omega$ should stay below an upper limit $\omega_{\max }$

$$
\omega_{\max }=\sqrt{\frac{F_{s t}}{m R_{s}}}=\sqrt{\frac{F_{s t}}{\rho s^{2} t R_{s}}}
$$

Where $R_{s}$ is the rotating arm length of the orbital shaker, $m$ is the mass of the part. The mass of a square part $m$ is equal to $\rho s^{2} t$, where $\rho / s / t$ are density/side length/thickness, respectively. For the $4 \mathrm{~mm}$ square PZT part, $\rho=7.8 \mathrm{~g} / \mathrm{cm}^{3}, s=4 \mathrm{~mm}, t=$ $190 \mu \mathrm{m}$.

We observed that alignment between PZT actuators and binding sites took place within 1 s, i.e., immediately after the orbital shaker $\left(R_{s}=1.27 \mathrm{~cm}\right)$ was turned on and ramped up from $0 \mathrm{rpm}$ to $200 \mathrm{rpm}$ (the shaking speed we set), so that $F_{s t}$ in (4) should be the surface tension on the PZT part when the part starts to leave the aligned state. According to [16], the maximum surface tension or restoring force by the acrylate-based adhesive on a 1-mm-square part leaving the aligned state is about $0.9 \times 10^{-4} \mathrm{~N}$. Due to the linear relation between surface tension and part side length, the maximum surface tension on the PZT part having $3 \mathrm{~mm}$ square hydrophilic bonding area is $F_{s t}=3 \times 0.9 \times 10^{-4} \mathrm{~N}=2.7 \times 10^{-4} \mathrm{~N}$. By plugging the values of $\rho / s / t / R_{s} / F_{s t}$ into (4), we get the shaking speed limit $\omega_{\max }=286 \mathrm{rpm}$, which is close to the experimental result $300 \mathrm{rpm}$, when the PZT part left the binding site.

\section{E. Electrical Connections by a Pressing Plate Method}

A pressing plate (Fig. 8) method is utilized to establish electrical connections between the PZT parts and the pump substrate (Fig. 9). The nonconductive adhesive can only provide mechanical bonding at the center of a PZT part. Because the PZT part is larger than the binding site and has an Au rim on the bonding face, electrical connections can be established through the $\mathrm{Au}$ rim. We apply a glass plate on the aligned PZT parts to squeeze out excess adhesive with a pressure of about $2 \times 10^{4} \mathrm{~N} / \mathrm{m}^{2}$, such that the Au rims touch the substrate [see Fig. 10(a)]. The PZT part has a surface with about $1 \mu \mathrm{m}$ roughness, which guarantees electrical connection even though the Au rim is coated with a thiol monolayer, and some adhesive residue may be trapped underneath it. Finally the electromechanical bonding is made permanent by polymerizing the adhesive on an $85^{\circ} \mathrm{C}$ hotplate for half an hour. The top electrodes of the PZT parts and the substrate are connected by wire bonding [see Fig. 10(b)]. Our later experimental tests show that all the 28 PZT actuators assembled 


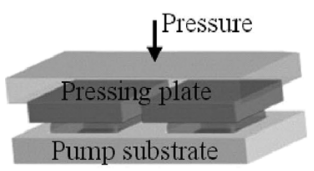

(a)

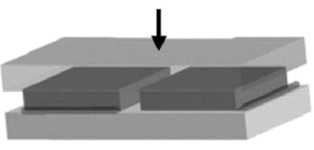

(b)

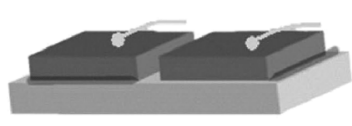

(c)

Fig. 8. Schematic overview of a pressing plate method to establish electrical connections at the rims of PZT parts: (a) a glass plate is introduced to press the aligned PZT parts; (b) excess adhesive is squeezed out of the wells and the rims of PZT parts contact the substrate; (c) adhesive is polymerized, the pressing plate is removed and the top electrical connections are made with solder bonding.

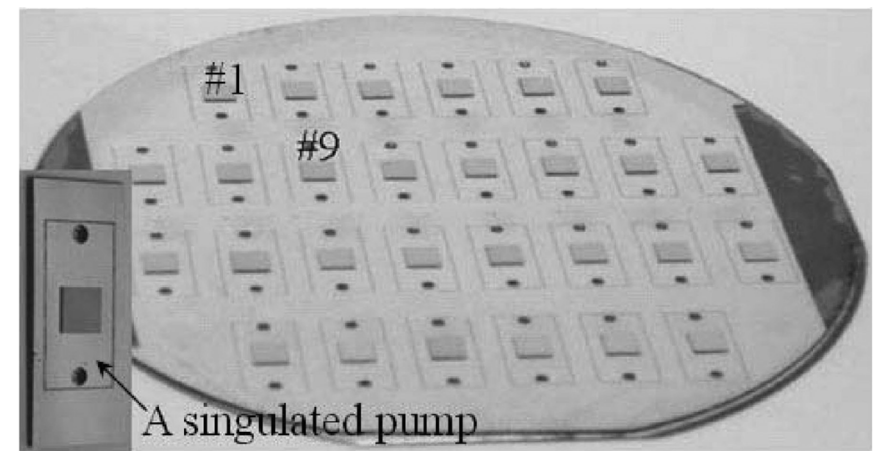

Fig. 9. Optical images of twenty-eight PZT actuators self-assembed on a $4^{\prime \prime}$ pump substrate and a singulated micropump.

on the 4 " pump substrate are electrically connected, which indicates a $100 \%$ yield for the pressing plate method.

\section{F. Adhesion Test}

A debonding test is performed in order to evaluate the bonding strength which is a key factor for PZT actuators. An experimental setup for the debonding test is schematically shown in Fig. 11: the bonded stack of substrate, cured adhesive and PZT actuator is held vertically with the edge of the PZT resting on a pushing tip attached to a digital scale. The digital scale reading for the total mass of all the items is zeroed out, and then a pressing force applied to the top of the bonded stack is increased until the PZT part is debonded, so the final reading in unit of kilograms multiplied by $g=9.81 \mathrm{~N} / \mathrm{kg}$ is the debonding force.

Hydrophilicity of binding sites, determined by interfacial energies, affects the bonding strength of PZT actuators. The binding sites for the above in-air assembly process are hydrophilic, while the binding sites were hydrophobic thiolated gold for our previously reported in-water assembly process [10]. In order to compare the bonding strength of these two assembly processes, we assemble eight 3-mm square PZT actuators (same bonding area as that in the above in-air assembly process) in a single batch with the same curable adhesive using the in-water assembly process. These eight PZT actuators are respectively debonded by forces of $8 \mathrm{~N}, 5 \mathrm{~N}, 7 \mathrm{~N}, 5 \mathrm{~N}, 9 \mathrm{~N}, 7$ $\mathrm{N}, 4 \mathrm{~N}$ and $6 \mathrm{~N}$. All the debonding processes take place at the interface between the cured adhesive and hydrophobic thiolated gold binding sites, which indicates the alkanethiol monolayer weakens the bonding. Five out of 28 PZT actuators assembled in air are randomly chosen for the debonding test, and none of them are debonded at the force of $\sim 20 \mathrm{~N}$, but the PZT edges contacting the pushing tip are broken.

\section{Pumping Tests}

\section{A. Resonant Frequencies}

To demonstrate performance uniformity of all the 28 pumps assembled simultaneously on the $4^{\prime \prime}$ substrate (Fig. 9), we use a laser vibrometer to measure their resonant frequencies without any fluid load. The frequency of the driving sinusoidal voltage signal is changed continuously from $1 \mathrm{kHz}$ to $150 \mathrm{kHz}$, and the maximum output of the laser vibrometer indicates a resonant frequency. The average of all the 28 fundamental resonant frequencies is $80.1 \mathrm{kHz}$, and the standard deviation is $2.0 \mathrm{kHz}$ (Fig. 12). The following factors may contribute to this $2.5 \%$ relative deviation: 1) PZT actuator tolerances of $\pm 50 \mu \mathrm{m}$ in length and width; 2) residual stress variance among the pump chamber diaphragms introduced by the anodic bonding process; 3 ) dimension variance among pump cavities in the silicon substrate due to non-uniformity of the DRIE processes (standard deviations of chamber depth and channel depth are, respectively, $0.55 \mu \mathrm{m}$ and $1.15 \mu \mathrm{m})$.

\section{B. Pumping Rates}

Eight pumps are chosen for pumping tests with ethanol on a reusable testing stage (Fig. 13). Each pump substrate is mounted onto the testing stage with silicone rubber rings. During the pumping tests, the inlet and outlet Teflon tubes (both tubes are about $15 \mathrm{~cm}$ long) are taped to a level workstation, and time is counted for the ethanol front end to move through a chosen 1 $\mathrm{cm}$ long region in the outlet tube. Because fluidic resistance of a tube increases with its length [17], the ethanol front end has a decreased speed as it moves towards the outlet tube end if the driving signal on the PZT is kept constant. To keep consistency between different driving frequencies, the ethanol front end is reset to the same initial position before applying a new driving frequency. The pumping results shown in Table II indicate: 1) the average and standard deviation of the fundamental resonant frequencies of the eight pumps are $6.99 \mathrm{kHz}$ and $0.10 \mathrm{kHz}$, respectively; 2) when the driving voltages are $90 \mathrm{~V}_{\mathrm{p}-\mathrm{p}}$, the average and standard deviation of the pumping rates at resonant frequencies are respectively $0.384 \mu \mathrm{l} / \mathrm{s}$ and $0.014 \mu \mathrm{l} / \mathrm{s}$.

\section{CONCLUSIONS AND Discussions}

We have demonstrated a capillary-driven self-assembly technique by assembling PZT actuators for 28 diffuser valve micropumps on a 4-inch substrate. All the 28 pumps work with high uniformity, which is indicated by small standard deviations of their resonant frequencies. This assembly technique has several major advantages over the conventional bonding method, manual bonding with highly viscous silver epoxy, for PZT actuators: precise placement by capillary-driven self-alignment [6], tilt free and strong bonding, well-controlled adhesive 


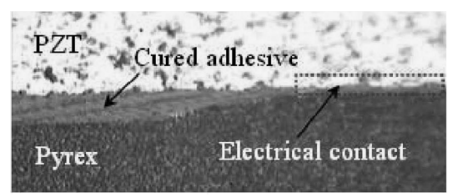

(a)

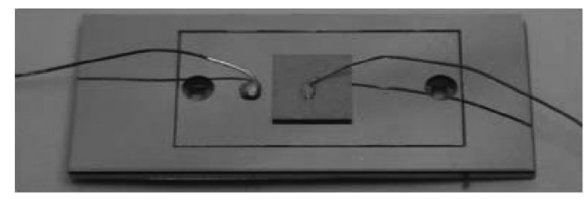

(b)

Fig. 10. Optical images showing the electrical connections to a PZT actuator: (a) a cross-section microscope image taken after a through cut of an assembled pump across the recessed pyrex binding site; (b) two thin electrical wires respectively solder bonded to the PZT top surface and the pump substrate, thus a driving voltage can be applied to activate the PZT actuator through these two leads.

TABLE II

Ethanol PumPing Results of THE EIght PUMPS

\begin{tabular}{|c|c|c|c|c|c|c|c|c|}
\hline Pump & 1 & 2 & 3 & 4 & 5 & 6 & 7 & 8 \\
\hline Fundamental Resonant Frequency $(\mathrm{kHz})$ & 7.0 & 7.0 & 6.8 & 6.9 & 7.0 & 7.0 & 7.1 & 7.1 \\
\hline Flow Rate at Resonant Frequency $(\mu \mathrm{l} / \mathrm{sec})^{a}$ & 0.387 & 0.408 & 0.365 & 0.385 & 0.365 & 0.390 & 0.385 & 0.386 \\
\hline
\end{tabular}

${ }^{a}$ Note: the driving voltage is $90 \mathrm{~V}_{\mathrm{p}-\mathrm{p}}$.

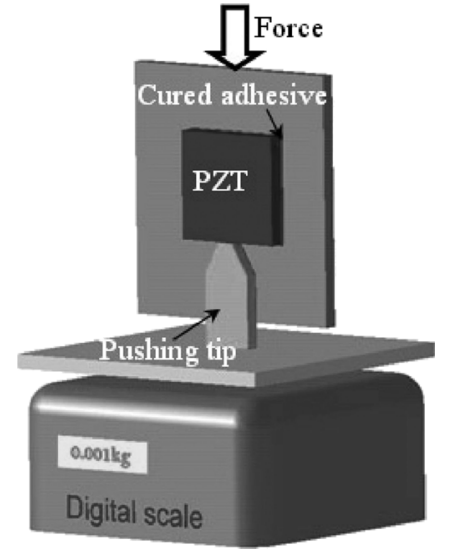

Fig. 11. Schematic experimental setup for debonding tests. A PZT actuator bonded to a substrate is pushed against a tip attached to a digital scale, and the applied force is increased and recorded until failure.

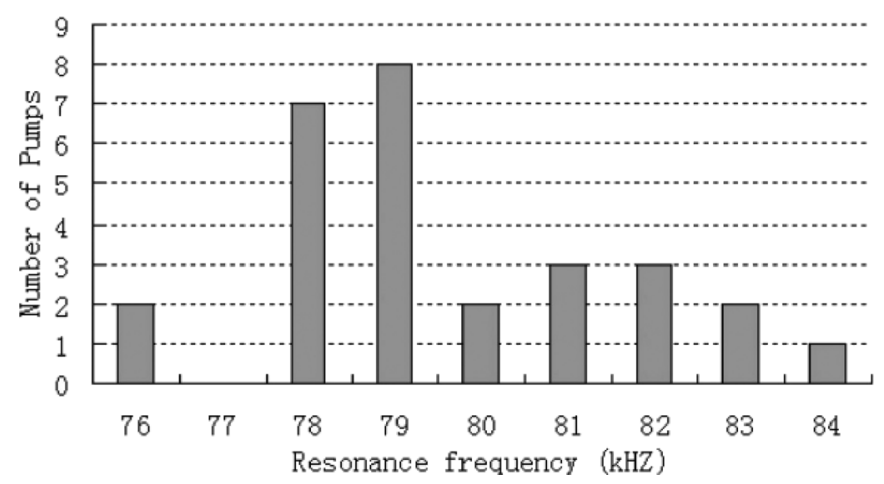

Fig. 12. Distribution of resonant frequencies for all the 28 pumps on a 4 -inch substrate.

thickness, electrical and mechanical connections established respectively at the rim and center of the assembled part, low process temperature.

Performance uniformity among the micropumps constructed in the same or different batches depends on both the fabrication steps for the pump substrates and the final assembly processes for PZT actuators. DRIE processes bring about various depths of the pump cavities even on the same substrate, and the anodic bonding process introduces different residual stresses on

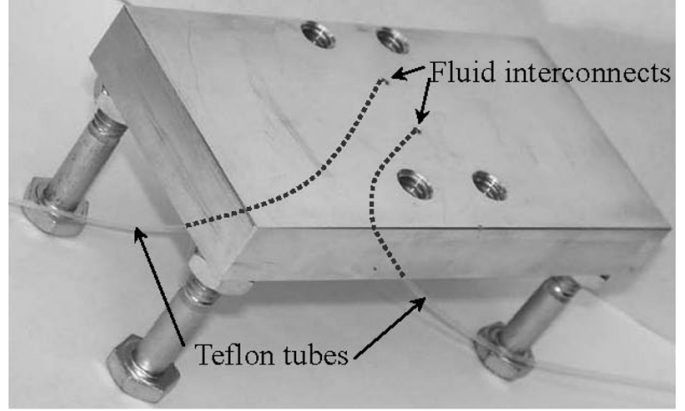

(a)

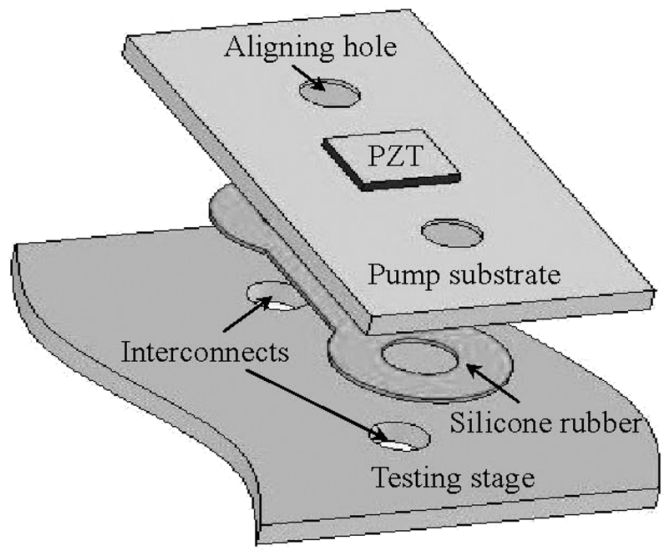

(b)

Fig. 13. A reusable testing stage: (a) an optical photograph of the stage before mounting a pump substrate; (b) a schematic exploded view of mounting a pump substrate with silicone rubber, which can be debonded without any residue by an appropriate shear force.

each pump diaphragm. The uniformity of DRIE processes relies on the improvement of the DRIE equipment and optimized process parameters. Low stress wafer bonding processes, e.g. low temperature anodic bonding [18], can improve the residual stress problem. Repeatability of the assembly process for PZT actuators relies on two major factors: recessed binding sites and placement of the PZT actuators. Recessed pyrex binding sites are formed by HF etching and the etching rate depends on the HF concentration. Provided good control of the HF concentration, the etching rate can be accurately controlled [19], [20], and so can the recessed depth for the binding sites. Accurate 
placement of the PZT actuators is achieved by the self-alignment process driven by surface tension forces, which has been shown to be highly repeatable.

This self-assembly process can also be applied to other types of electromechanical microchips. Especially for microchips with materials or structures sensitive to aqueous assembly environments, this dry assembly method shows a significant advantage over other wet assembly techniques. With batch process capabilities, this self-assembly technique supports construction of more versatile and miniaturized microsystems.

\section{ACKNOWLEDGMENT}

The authors would like to thank F. Forster and Y. Hanein for inspiring discussions, B. Williams and C. Morris for helping with the pump design and pumping tests, T.-C. Chen for helping with laser cutting, and Y. Wang and X. Xiong for helpful comments and discussions.

\section{REFERENCES}

[1] F. K. Forster, R. L. Bardell, M. A. Afromowitz, N. R. Sharma, and A. Blanchard, "Design, fabrication and testing of fixed-valve micropumps," in Proc. ASME Fluids Eng. Div., ASME Int. Mech. Eng. Congr. Expo., 1995, vol. 234, pp. 39-44.

[2] Z. Yang, H. Goto, M. Matsumoto, and R. Maeda, "Ultrasonic micromixer for microfluidic systems," in Proc. IEEE Conf. Micro Electro Mech. Syst. (MEMS'2000), 2000, pp. 80-85.

[3] L.-S. Jang, D. R. Meldrum, and M. R. Holl, "An active mixer for microscale DNA purification and sequencing reaction clean-up," in Proc. 6th Int. Conf. Mini. Chem. Biochem. Anal. Syst. ( $\mu$ TAS'02), Nara, Japan, 2002, pp. 239-241.

[4] A. Kuoni, M. Boillat, and N. de Rooij, "A modular high density multichannel dispenser for micro-array printing," in Proc. 12th Int. Conf. Solid-State Sens. Actuators (Transducers'03), 2003, pp. 372-375.

[5] H. Jacobs, A. Tao, A. Schwartz, D. Gracias, and G. Whitesides, "Fabrication of cylindrical display by patterned assembly," Science, vol. 296, pp. 323-325, 2002.

[6] U. Srinivasan, M. H. Helmbrecht, C. Rembe, R. S. Muller, and R. T. Howe, "Fluidic self-assembly of micromirrors onto microactuators using capillary forces," IEEE J. Sel. Topics Quantum Electron., vol. 8, no. 1, pp. 4-11, 2002.

[7] R. Syms and E. M. Yeatman, "Self-assembly of three-dimensional microstructures using rotation by surface tension forces," Electron. Lett., vol. 29, no. 9, pp. 662-664, 1993.

[8] K. F. Harsh, V. M. Bright, and Y. C. Lee, "Solder self-assembly for three-dimensional microelectromechanical systems," Sens. Actuators A, Phys., vol. 77, no. 3, pp. 237-244, 1999.

[9] R. R. A. Syms, E. M. Yeatman, V. M. Bright, and G. M. Whitesides, "Surface tension-powered self-assembly of microstructures-the state-of-the-art," J. Microelectromech. Syst., vol. 12, no. 4, pp. $387-417,2003$.

[10] X. Xiong, Y. Hanein, J. Fang, Y. Wang, W. Wang, D. T. Schwartz, and K. F. Böhringer, "Controlled multi-batch self-assembly of micro devices," J. Microelectromech. Syst., vol. 12, no. 2, pp. 117-127, Apr. 2003.

[11] K. L. Scott, R. T. Howe, and C. J. Radke, "Model for micropart planarization in capillary-based microassembly," in Proc. 12th Int. Conf. Solid-State Sens. Actuators (Transducers'03), 2003, pp. 1319-1322.

[12] B. E. Williams and F. K. Forster, "Micropump design of optimum pressure/flow characteristics," in Proc. MEMS-3 ASME Int. Mech. Eng. Congr. Expo. (New York), 2001.

[13] A. Olsson, G. Stemme, and E. Stemme, "A numerical design study of the valveless diffuser pump using a lumped mass model," J. Micromech. Microeng., vol. 9, pp. 34-44, 1999.

[14] M. Heschel, M. Muellenborn, and S. Bouwstra, "Fabrication and characterization of truly 3-d diffuser/nozzle microstructures in silicon," $J$. Microelectromech. Syst., vol. 16, no. 1, pp. 41-47, 1997.

[15] K. Yang, I. Chen, B. Shew, and C. Wang, "Investigation of the flow characteristics within a micronozzle/diffuser," J. Micromech. Microeng., vol. 14, pp. 26-31, 2004.
[16] A. Greiner, J. Lienemann, J. G. Korvink, X. Xiong, Y. Hanein, and K. F. Böhringer, "Capillary forces in micro-fluidic self-assembly," in Proc. 5th Int. Conf. Modeling Simulation Microsyst. (MSM'02), 2002, pp. $198-201$.

[17] C. J. Morris and F. K. Forster, "Low-order modeling of resonance for fixed-valve micropumps based on first principles," J. Microelectromech. Syst., vol. 12, no. 3, pp. 325-334, 2003.

[18] J. Wei, H. Xie, M. L. Nai, C. K. Wong, and L. C. Lee, "Low temperature wafer anodic bonding," J. Micromech. Microeng., vol. 13, pp. 217-222, 2003.

[19] I. Kashkoush, E. Brause, R. Novak, and R. Grant, "In situ chemical concentration control for wafer wet cleaning," in Proc. Mater. Res. Soci. Symp., 1997, vol. 477, pp. 311-316.

[20] I. Kashkoush, G. Chen, P. Boelen, and M. Geomini, "Hf concentration control in ic manufacturing," in Proc. 6th Int. Symp. Cleaning Technol. Semicond. Device Manufact., Honolulu, HI, 1999.

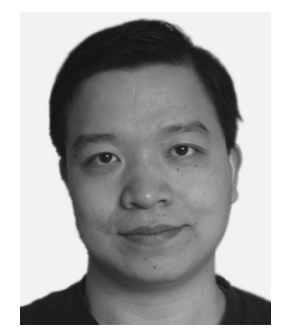

Jiandong Fang received the Bachelor's degree in physics from the University of Science and Technology of China in 1999 and the Master's degree in physics from the University of Washington, Seattle, in 2001. He is currently pursuing the Ph.D. degree in electrical engineering of the University of Washington.

His research interests include microfluidic pumps, self assembly, and microdevice packaging.

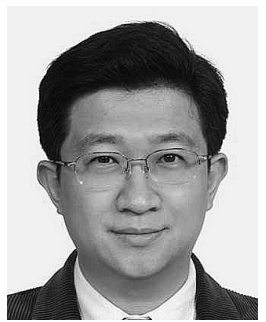

Kerwin Wang received the B.S. degree in mechanical engineering from Chung Yuan Christian University, Taiwan, the M.S. degree in mechanical engineering from the State University of New York at Buffalo, NY, and the Ph.D. degree in electrical engineering in 2004 from the University of Washington, Seattle.

During his dissertation work on micro-display modules, he invented, modeled, and tested zigzag actuators and floating sliders; he also held an internship from Microsoft Research at Redmond, WA. Upon completion of his Ph.D. program, he spent a year as a Postdoctoral Researcher at the University of Washington, where he investigated micro/nano-assembly techniques. In July of 2005, he was hired as a Visiting Postdoctoral Researcher at the Intel Component Research Group and developed high-packing-density micro-assembly for solid-state cooling applications.

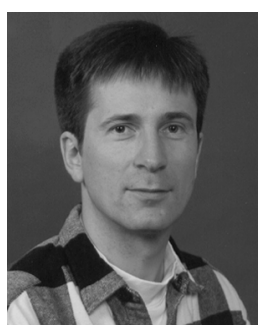

Karl F. Böhringer (S'94-M'97-SM'03) received his Dipl.-Inform. degree from the University of Karlsruhe, Germany, in 1990 and the M.S./Ph.D. degrees in computer science from Cornell University, Ithaca, NY, in 1993-1997.

He was a Visiting Scholar at Stanford University in 1994-1995 and a Postdoctoral Researcher at the University of California, Berkeley from 1996 to 1998. He joined the Electrical Engineering department at the University of Washington in Seattle, WA, in 1998 where he is currently Associate Professor. He also held visiting faculty positions at the Universities of Tohoku, Tokyo, Kyoto, Japan, and São Paulo, Brazil. His research interests include microelectromechanical systems (MEMS), manipulation and assembly from macroto nanoscales, microfluidic systems for the life sciences, and microrobotics. He has created, among others, multibatch selfassembling systems, massively parallel microactuator arrays, and a walking microrobot.

Dr. Böhringer is member of the Society for Nanoscale Science, Computing and Engineering (ISNSCE), the American Society for Engineering Education (ASEE), and the German Society for Information Sciences (GI). He was awarded a Long-term Invitational Fellowship for Research in Japan by the Japan Society for the Promotion of Science (JSPS) in 2004, an IEEE Robotics \& Automation Society Academic Early Career Award in 2004, an NSF CAREER Award in 1999, and an NSF Postdoctoral Associateship in 1997. His work was listed among the "Top 100 Science Stories of 2002" in Discover magazine. $\mathrm{He}$ is an associate editor of ASME/IEEE JOURNAL OF Microelectromechanical SyStems and IEEE TRANSACTIONS ON AUTOMATION SCIENCE AND ENGINEERING and has served, among others, on technical program committees for the IEEE MEMS and Transducers conferences. 